\title{
Pre-filled Pen Syringe
}

National Cancer Institute

\section{Source}

National Cancer Institute. Pre-filled Pen Syringe. NCI Thesaurus. Code C97717.

A syringe that lacks a conventional plunger, resembles a writing pen, and is designed to dispense a pre-loaded dose of a drug. It may be designed to deliver a single dose or be designed for repeated use. 